\title{
Characterization of Welding Discontinuities by Combined Phased Array Ultrasonic and Artificial Neural Network
}

\author{
Ahmed Mazloum $^{a}$, Ahmed Mahrous $^{a}$, Alber Alphonse Sadek $^{b}$, Abd ElRahman Mohamed Moussa $^{a}$ \\ (a) Mechanical Engineering Department, Helwan University, Helwan, Egypt. \\ (b) Edison Welding Institute, EWI, Ohio, USA.
}

\begin{abstract}
Automatic inspection of welded gas pipelines is desirable because human inspectors are not always consistent evaluators. In addition, automatic inspection decreases the cost of inspection process and improves the inspection quality. In this paper, a proposed system named Phased Array for Characterizing Discontinuities "PACD" which combined 2D S-scan images into 3D images (volumetric scan) is more reliable and accurate and easily interpretation. The system is checking the shape, location, width and length of each discontinuity and the echo width and height of the A-scan. After that PACD inputs them to learned artificial neural network (ANN) to characterize 12-types of common welding discontinuities in gas pipelines welded by shield metal arc welding (SMAW). After verification the PACD system can be easily characterized welding discontinuities types with zero error.
\end{abstract}

Keywords : Welding discontinuities, Phased Array, ANN.

\section{Introduction \& Problem Description}

The control of welded joints in many industries such as the nuclear, naval, aviation, oil and gas plants particularly important for critical applications. Where weld failure can be catastrophic, such as in pressure vessels, load-bearing structural members, power plants, pipe lines (P/Ls). However, due to the complexity involved in welding process choices and procedures, the joints which having weld defects like (porosity, undercuts, blow holes, slag inclusion, incomplete penetration, lack of side wall fusion and excess penetration during the joining process, hence, weld quality inspection and qualification of these fabricated components. Most of welded joints failures are due to inaccurate interpretation (determination, sizing, measuring and evaluation) of their defects.

So, the purpose of the automation of the discontinuity's classification is to reduce the interpretation time and eliminate the subjective aspect in the analysis done by the inspector, this way increasing the reliability in the inspection. In this sense, the combination of S-Scan images processing with artificial neural networks (ANN) have a fundamental role in a system of automatic classification of S-scan images of welded joints. 


\section{Previous Work}

Over the last two decades, there has been a considerably increase in the number of publications of research projects for the detection and classification of welding discontinuities in radiographs/ultrasonic tests using image processing and pattern recognition tools. A classification system as such would allow a reduction in some inherent inexactness's that occur in the conventional method, consequently increasing the precision of the report [1].

The first theory that suggests Algorithms and neural networks was initiated by Hopgood \& Woodcock [2] to build a suitable software framework for image interpretation, and to use this framework to interpret automatically ultrasonic B-Scan images of components and characterize any defects therein. In Ref. [3], a methodology was developed to detect defects obtained from ultrasonic-based NDT using the multilayer perceptrons (MLP) Neural Network. The authors found that results obtained by using discrete wavelet transform (for feature extraction) and neural networks were superior over the classification of NDT signals using only neural networks. In Ref. [4], the authors classified three kinds of welding defects using Time Of Flight Diffraction (TOFD) technique based on signal processing techniques and artificial neural network. The Fourier transform and wavelet transform were used for preprocessing A-Scan signals. They implemented linear pattern classifiers into the network. In comparison with Fourier transform, Wavelet transform results in better classification.

In Ref. [5], the wavelet transform has been successfully used in experiments to suppress noise and enhance flaw location from ultrasonic signals, with a good defect localization. The obtained result was then feed to an automatic Artificial Neural Network classifier to classify welding defects from A-scan data. In Ref. [6], the authors evaluated the application of ANN for pattern recognition of ultrasonic signals in weld defects using pulse echo and TOFD techniques. They classified four welding defects by using supervised feed forward back propagation type neural network. They reported the success rate of $72.5 \%$ for pulse echo and $77.5 \%$ for TOFD technique, both without preprocessing. The geometry of a weld is an important factor for determining which angle to be used for inspection in ultrasonic testing. The phased array sectorial scan makes it possible to scan areas with restricted access and also enables angles to be changed according to the geometry of the weld under inspection. Phased array systems allow inspection angles to be adjusted accurately and modified easily so as to obtain better response from defects [7].

So, the objective of this paper to develop :

Phased Array results in 3D instead of 2D, to improve the characterization of welding discontinuities (Volumetric Scanning). 
An artificial intelligent model ANN to characterize arc welding discontinuities. Automatic system for detecting discontinuity type to eliminate Human error.

\section{Proposed System}

Fig.1 shows the equipment and instrumentation, that used in the experiment, and it consists of the following :

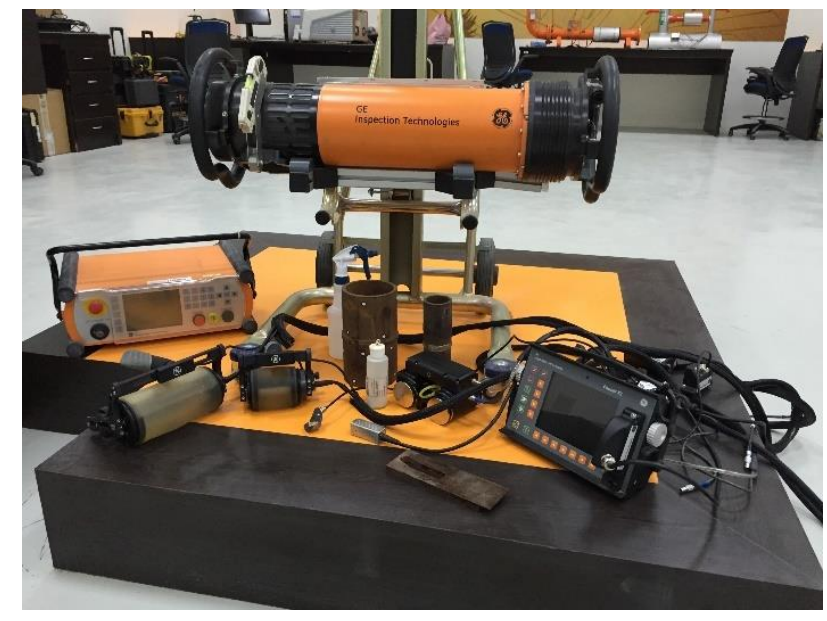

Fig.(1). Photo RT\&UT Equipment used.

\subsection{For radiography test (RT)}

- X-Ray BHGE-Generator - Eresco MF4 160kv,

- Computerized Radiography (CR) System : CRxFlex,

- CR Phosphor image plates IPC2, IPS with different sizes $10 \times 24 \& 10 \times 40 \mathrm{~cm} \&$ $10 \times 48 \mathrm{~cm}$,

- Rhythm Software.

\subsection{For ultrasonic test (UT)}

- Phased Array KRAUTKRAMER "Phasor-XS" unit (BHGE Inspection Technologies),

- Different Probes/wedges $2-4 \mathrm{MHz}\left(35^{\circ}-75^{\circ}\right)$ with 8-64 elements,

- RotoArray probes $(5 \mathrm{MHz} / 64$ element $/ 0.8 \mathrm{~mm}$ pith $\times 6.4 \mathrm{~mm}$ width),

- RotoArray probes $(5 \mathrm{MHz} / 64$ element $/ 1.27 \mathrm{~mm}$ pith $\times 8 \mathrm{~mm}$ width),

- PATH Scanner and Alstom Inspection robotics,

- V1, V2, Stepped, AWS Resolution and PA-DM METRED Calibration blocks,

- Gel and Water is used as a couplant.

\subsection{Welding joints}

Figs.(2 and 3) show a photograph for the different specimens used. And all listed in table contains the profile and number of defects. The program of this research work was conducted in two different laboratories, i.e. two groups A and B. The first group 
(A) was chosen from PETROJET and MASSA workshops and different projects sites, representing a wide range of welding defects. The second group (B), was prepared at Abdullah-Fouad Application center. In this group the welding parameters were monitored, for several welding defects.
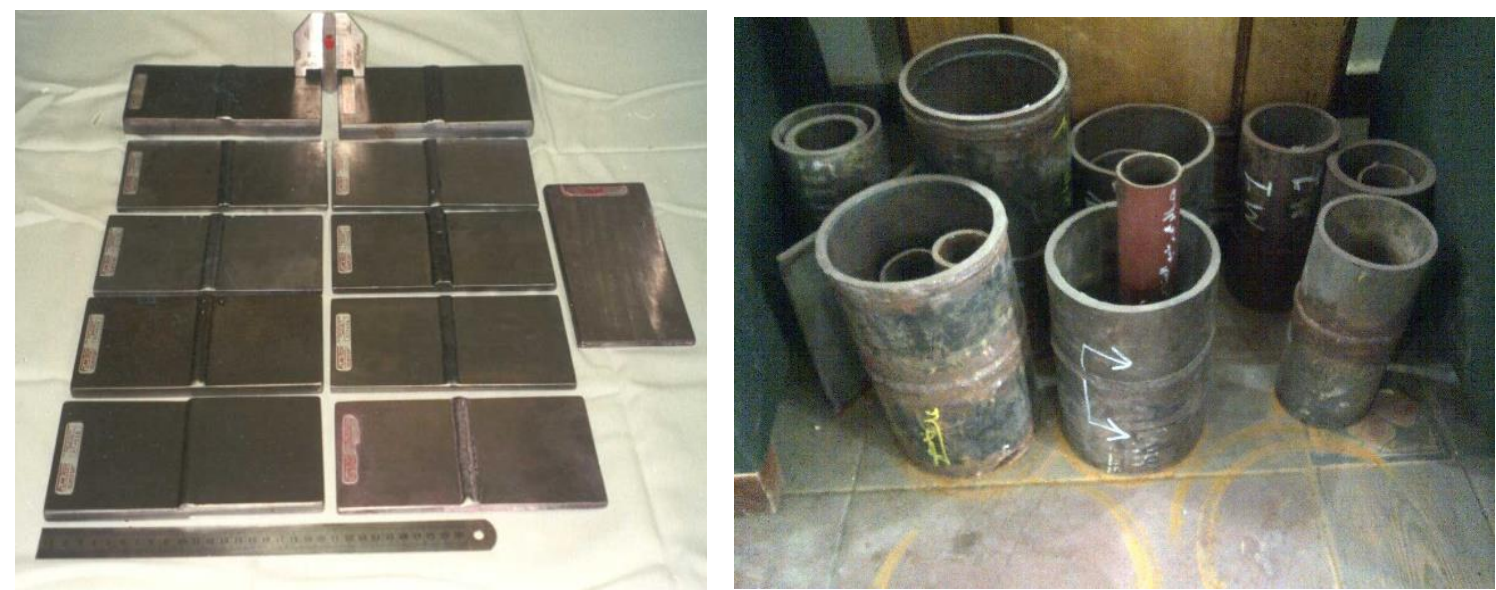

Fig.(2) Photograph of group(A): PETROJET\&MASSA butt welded joints with standard \& Practical Discontinuities.
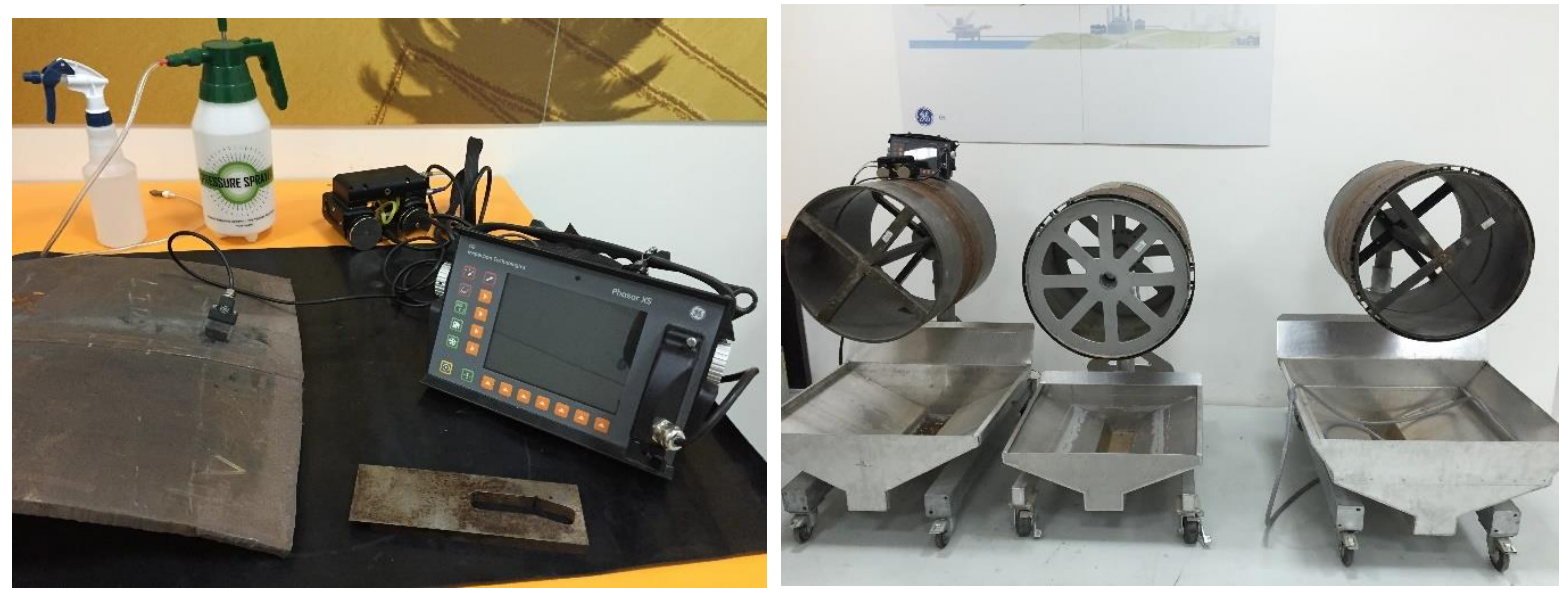

Fig.(3) Photograph of group (B): Abdullah Fouad's butt welded joints.

The welding joint direction was determined by measuring horizontally along the $\mathrm{X}$ axis from the starting point (left end) of the material to the starting point of the discontinuity and then by measuring vertically along the Y-axis from the center of the weld bead (either positively or negatively). The discontinuity length was then measured from the beginning to the end point in the $\mathrm{X}$-axis direction and the width from the same points but in the $\mathrm{Y}$-axis direction. The defect depth was measured from the top surface towards the beginning of the discontinuity in the Z-direction. As shown in Fig.(4). 


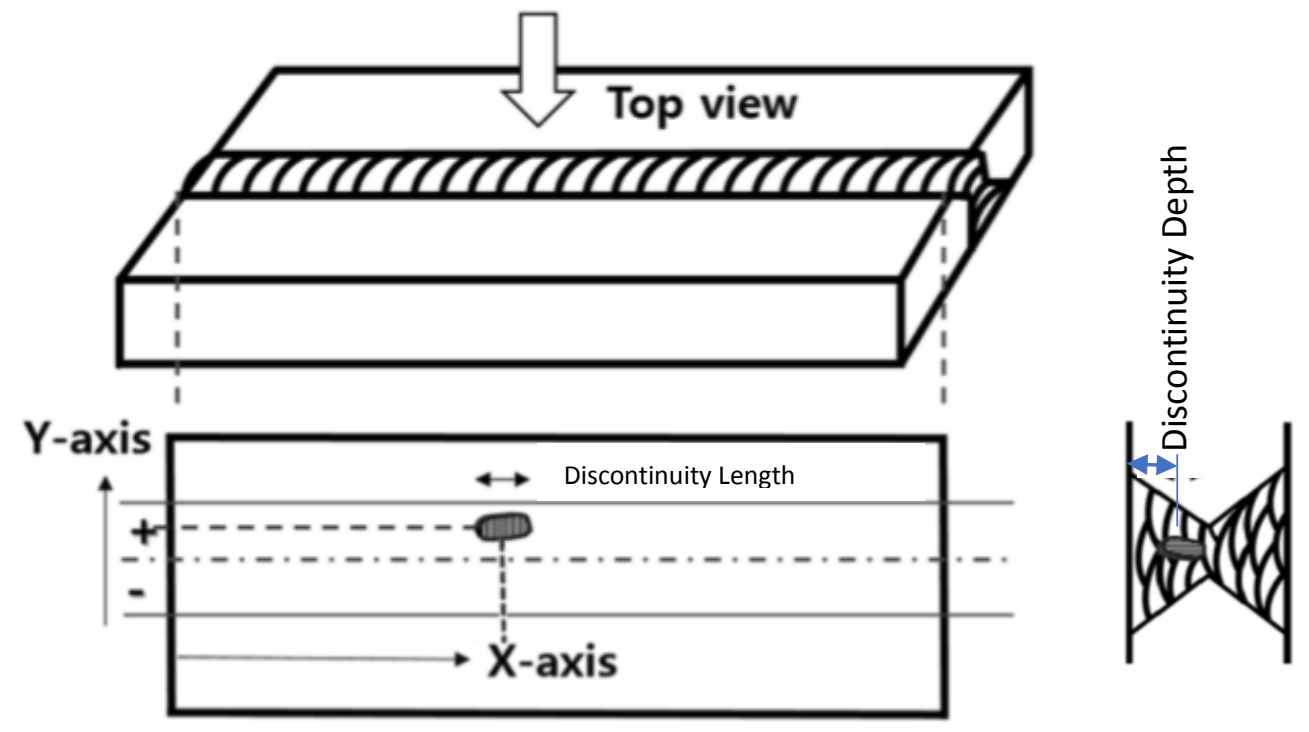

Fig.(4). Position, Length, width and depth of welding discontinuity.

\section{Image Processing}

The union of pictures from different S-scans is materialized as 3D images. Its recognizable to estimate the discontinuities characterizations as below :

\subsection{Estimating the discontinuity location from S-scan images}

Fig. (5) shows the discontinuities position measured as Position Vertical (Z-Direction) (PV), Position Horizontal (Y-Direction) (PH), Position Longitudinal (X-Direction) (PL) and we can calculate discontinuity Width (W). Fig. (6) shows the classification of discontinuities in Y-direction as Horizontal Side (HS), Horizontal Medium (HM) \& Position Medium (PM).

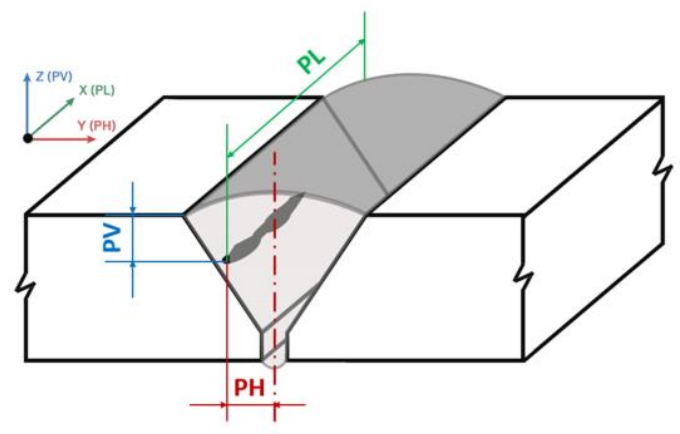

Fig. (5) Discontinuity Location

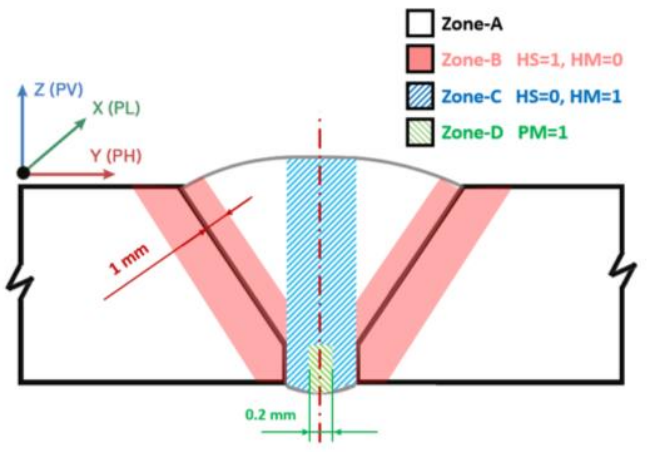

From Fig. (6). Fig.(6) Classification of welding If; The discontinuities Y-Direction 
location depends on PV \& PH locate on :

(Range-A) ,", that means ,, $\mathrm{HS}=1 \& \mathrm{HM}=0$

(Range-B) „, that means ,, $\mathrm{HS}=0$ \& $\mathrm{HM}=1$

(Range-C) „, that means ,, $\mathrm{PM}=1$

Also, Fig. (7) shows the classification of discontinuities in Z-direction as Vertical High (VH), Vertical Medium (VM) and Vertical Low (VL).

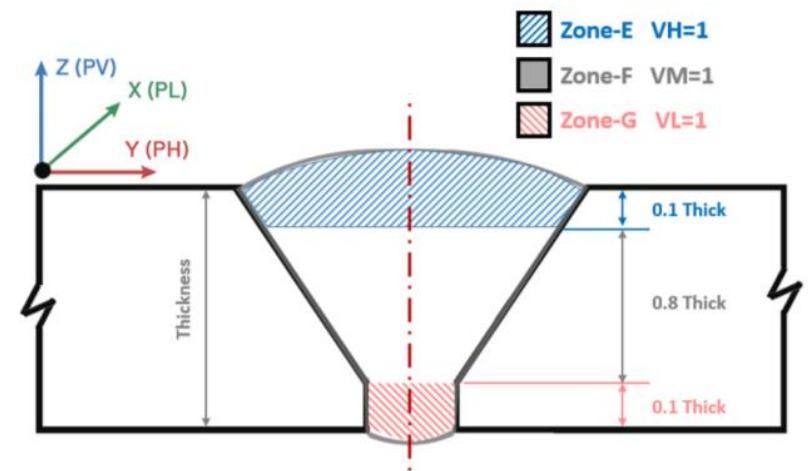

Fig.(7). Classification of welding discontinuities Z-Direction.

If; The location depends on PV locate on :

Range (D) ,", that means ,, $\mathrm{VH}=1, \mathrm{VM}=0 \& \mathrm{VL}=0$

Range (E) ,, that means ,, $\mathrm{VH}=0, \mathrm{VM}=1 \& \mathrm{VL}=0$

Range $(\mathrm{F})$, , that means ,, $\mathrm{VH}=0, \mathrm{VM}=0 \& \mathrm{VL}=1$

\subsection{Estimating the discontinuity shape from C-scan}

After union of pictures from S-Scan it can be materialized as 3D image and can estimate the C-Scan form which can be found the shape of discontinuity and calculate the discontinuity length (L), Largest discontinuity width (LW), Area of discontinuity $\left(A_{r}\right)$, Perimeter, Minimum Boundary box area $\left(A_{\min }\right)$. As shown on Fig.(8).

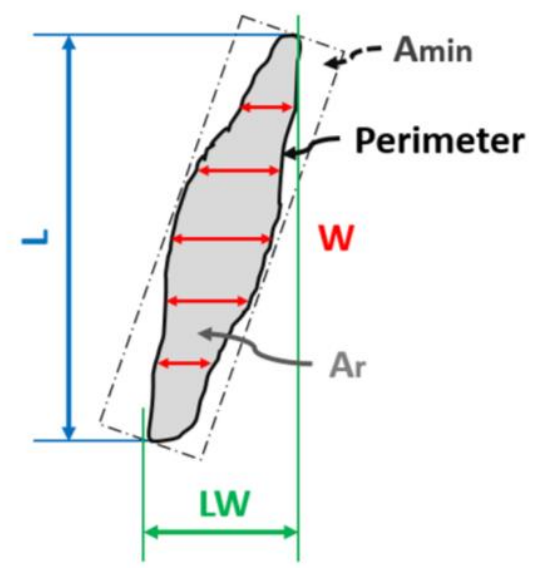

Fig.(8). C-Scan estimated from materialization process. 
Discontinuities shape classified into three categories, which are circular (C), rectangular (R) and irregular (I). Two factors were introduced to identify the shape regions. The first factor called form factor $\left(\boldsymbol{F}_{\boldsymbol{f}}\right)$ and is used to measure the circularity of regions based on its area and perimeter. The form factor $\boldsymbol{F}_{\boldsymbol{f}}$ is calculated using Eq.(1).

$$
F_{f}=\frac{(\text { Perimeter })^{2}}{4 \pi \text { Area }}
$$

For circular regions, the form factor $\left(\boldsymbol{F}_{\boldsymbol{f}}\right)$ is equal to 1.0. Founded that; difficult to obtain a value of 1.0 for the form factor. Therefore, a tolerance value $\pm 10 \%$ is used to identify the circular discontinuities.

$$
\begin{array}{lll}
\text { If; } \quad \begin{array}{l}
F_{f}=0.9 \text { to } 1.1 \\
F_{f}=<0.9 \text { or }>1.1
\end{array} \quad \text { that means } & \mathrm{C}=1, \mathrm{R} \& \mathrm{I}=0 \\
& \text { theans } & \mathrm{C}=0, \mathrm{R} \& \mathrm{I}=1 \text { or } 0
\end{array}
$$

The second factor used to identify the shape of discontinuities called rectangularity factor $\left(R_{f}\right)$. It is used to measure the rectangularity of regions based on the area of the region and the area of the minimum rectangle enclosing it. The rectangularity factor $R_{f}$ is calculated by Eq.(2).

$$
R_{f}=\frac{A_{r}}{A_{\min }} \quad \text { Eq. }(2)
$$

Where calculated area $\left(A_{r}\right)$ is the calculated area of the discontinuity and $\left(A_{\min }\right)$ is the area of the minimum rectangle that encloses the discontinuity. The $R_{f}$ is equal to 1.0 for actual rectangular regions.

To identify the shape of the discontinuity, the form factor $\left(\boldsymbol{F}_{\boldsymbol{f}}\right)$ is calculated first. If $\boldsymbol{F}_{\boldsymbol{f}}$ lies between 0.90 and 1.10 , the discontinuity shape is considered circular, otherwise, the rectangularity factor $\left(\boldsymbol{R}_{\boldsymbol{f}}\right)$ is determined. If $\boldsymbol{R}_{\boldsymbol{f}}$ lies between 0.9 and 1.10 , the discontinuity shape is considered rectangular, else it is considered irregular.

$$
\text { If; } \begin{array}{lll}
R_{f}=0.9 \text { to } 1.1 \quad \text { that means } & \mathrm{C}=0, \mathrm{R}=1 \& \mathrm{I}=0 \\
& R_{f}=<0.9 \text { or }>1.1 \quad \text { that means } & \mathrm{C}=0, \mathrm{R}=0 \& \mathrm{I}=1
\end{array}
$$

The orientation of each discontinuity is calculated based on its length and width. If the length (size in X-Direction) is greater than the width (size in Y-Direction), the orientation is considered Longitudinal (Long. = 1); otherwise it is considered Transverse (Long. $=0$ ) as shown on Fig.(9). Width variation (o) : 
If $o: 0.5$ that means parameter Sigma $=1$

Otherwise Sigma $=0$

\subsection{Estimating the UT echo width (EW)/height (EH)}

Moreover, the information of (EW) \& (EH) taken from the A-Scan images which taken from the "Phasor-XS" device as shown in Fig.(9) also used to identify discontinuities detection. The setup is the optimal to receive the highest amount of reflected energy from the flank.

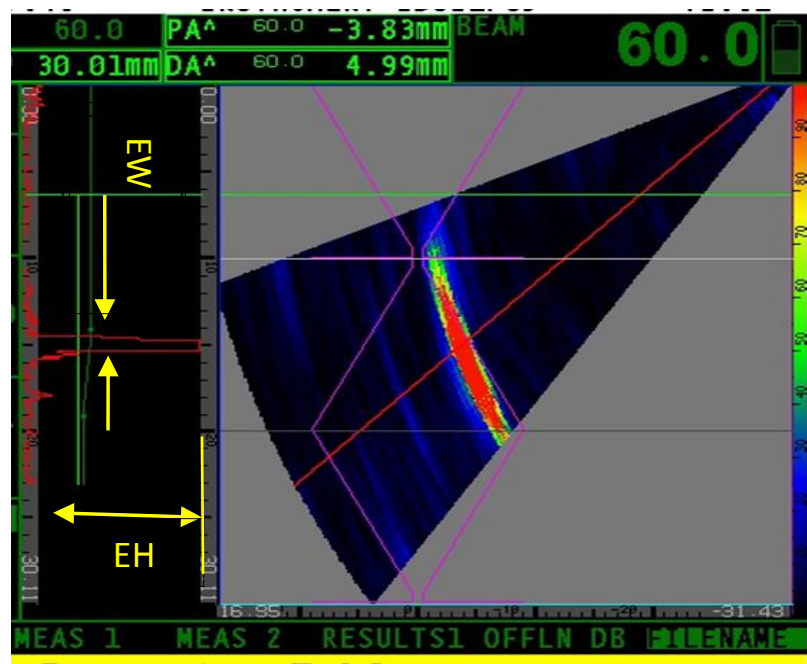

Fig.(9). Classification of Echo Width (EW)/Hight (EH) from Phasor-XS device.

If; EW $\geq 4.5$ (average) ,,, that means ,, Parameter Echo Width (PEW) $=1$

Otherwise $\mathrm{PEW}=0$

If; EH $\geq 0.9$ FSH (Full Screen Hight) ,,, that means ,, Parameter Echo Hight $(\mathrm{PEH})=$ 1

Otherwise $\mathrm{PEH}=0$

\subsection{Estimating all data collected}

Summarized all the data collected from different S-Scan and 3D images as mentioned into Table (1) :

Table (1). Data from S-Scan and 3D images.

\begin{tabular}{|c|c|}
\hline \multirow{6}{*}{ 2D } & $\begin{array}{l}\mathbf{P V} \text { (Position Vertical) (Z-Direction) } \\
\end{array}$ \\
\hline & PH (Position Horizontal) (Y-Direction) \\
\hline & 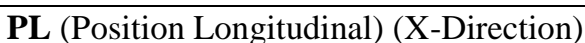 \\
\hline & W (Discontinuity Width) \\
\hline & EH (Echo Height) \\
\hline & EW (Echo Width) \\
\hline \multirow{4}{*}{ 3D } & L (Lenght) \\
\hline & LW (Largest Width) \\
\hline & $A_{\min }$ (Minimum Boundary Box Area) \\
\hline & $A_{r}$ (Area of Discontinuity) \\
\hline
\end{tabular}




\begin{tabular}{|l|l|}
\hline & Perimiter \\
\hline \multirow{3}{*}{ Calculate } & $F_{f}$ (Form Factor) \\
\cline { 2 - 2 } & $R_{f}$ (Rectangularity Factor) \\
\hline
\end{tabular}

Table (2) mention the parameters which calculated and its data collected which depend on :

Table (2). Parameters and all the data depend on.

\begin{tabular}{|l|l|l|}
\hline Parameters & Data depends on \\
\hline HS (Horizontal Side) & PV (Position Vertical), PH (Position Horizontal) \\
\hline HM (Horizontal Medium) & PV (Position Vertical), PH (Position Horizontal) \\
\hline VH (Vertical High) & PV (Position Vertical) \\
\hline VM (Vertical Medium) & PV (Position Vertical) \\
\hline VL (Vertical Low) & PV (Position Vertical) \\
\hline C (Circular) & $F_{f}$ (Form Factor) & $0.9<F_{f}<1.1$ \\
\hline R (Rectangular) & $R_{f}$ (Rectangularity Factor) & $0.9<R_{f}<1.1$ \\
\hline I (Irregular) & $\mathbf{C}$ (Circular), R (Rectangular) & $\mathrm{C} \& \mathrm{R}=0$ \\
\hline Long. (Longitudinal) & (Length/width) $>1.1$ & If $>0.5$ that mean $o=1$ \\
\hline Sig (variation) & O' (w) & $\begin{array}{l}\text { If }-0.1<\text { mean }(\mathrm{PH})<+0.1 \mathrm{~mm} \text { that mean } \\
\text { PH }=1 \text { Otherwise PH }=0\end{array}$ \\
\hline $\begin{array}{l}\text { PM (Position Medium) } \\
\text { PEW (Parameter Echo } \\
\text { Width) }\end{array}$ & $\mathbf{P H}$ (Position Horizontal) & At max.(EH) \\
\hline PEH (Parameter Echo Hight) & EH (Echo Hight) & \\
\hline
\end{tabular}

\section{Results of Image Processing}

Taken two types of discontinuities [Lack of Pen. (LOP) and Long. Crack] as an example of applying 3-D images from different types of views (Top, Front \& Side).

\subsection{Lack of penetration (LOP)}

As shown in Fig.(10) that LOP is considered as longitudinal discontinuity at the middle of the weld root and Fig.(11) shows different S-Scan images taken from XAxis direction every $1 \mathrm{~mm}$ length position.

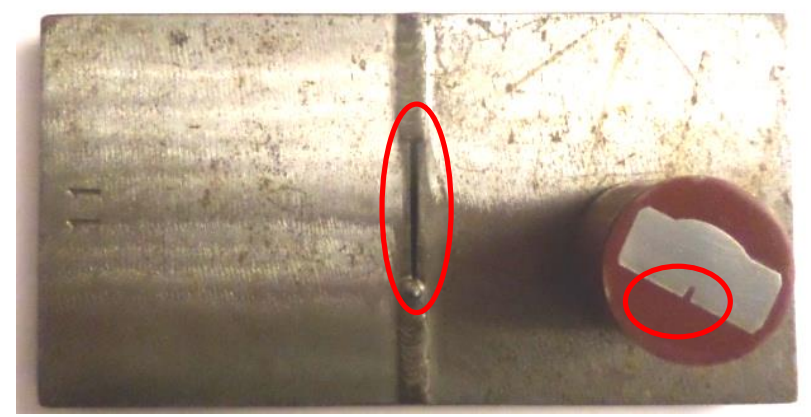

Fig.(10). Photo of LOP standard Discontinuity. 
LOP can be found exactly at the middle of weld root, and it can be characterized as longitudinal and rectangular shape, But the standard division of discontinuity width was low, the echo width was low and the echo height was large (Sharp).

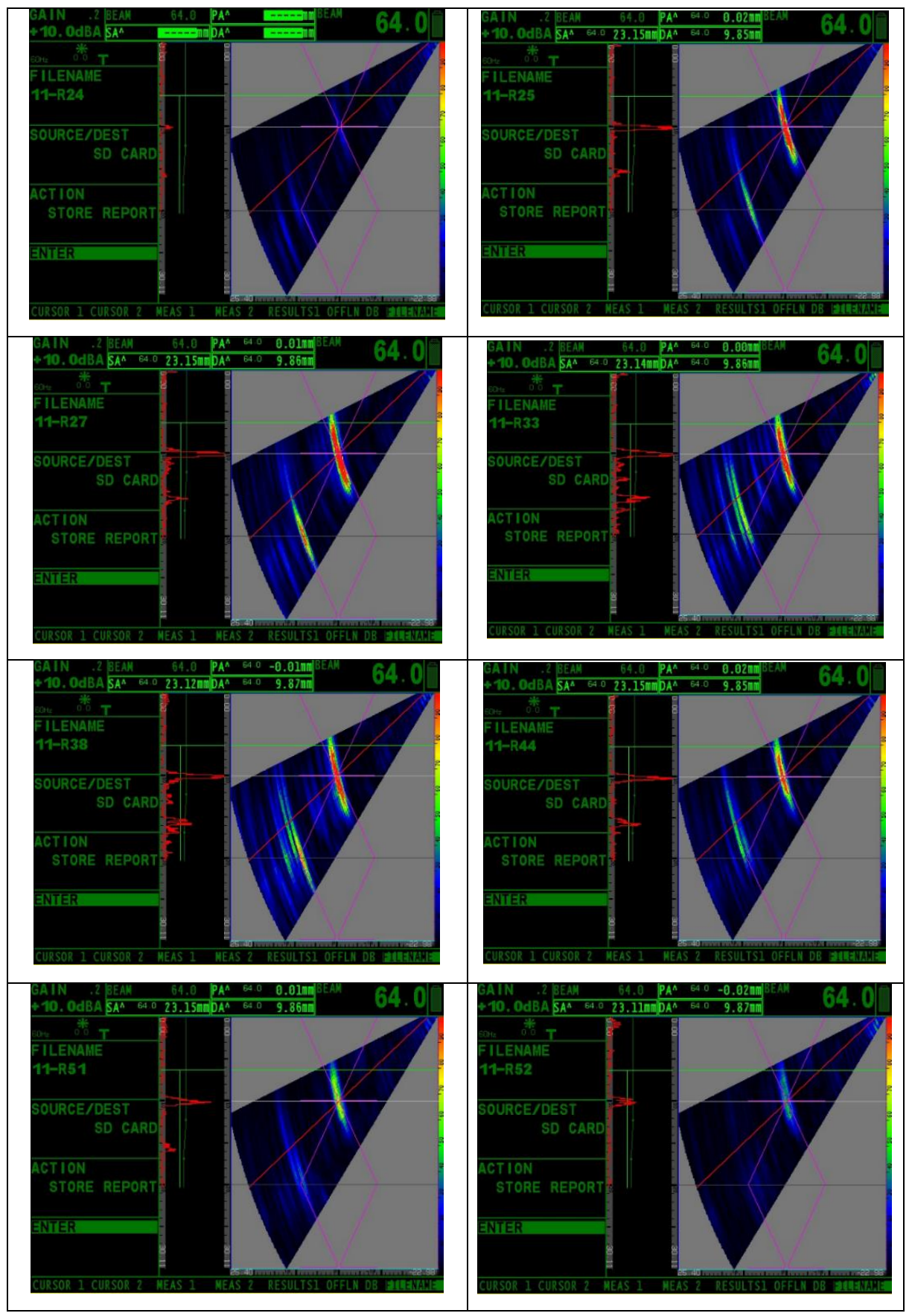

Fig.(11). LOP S-Scans taken respectively to be input to MATLAB Program. 


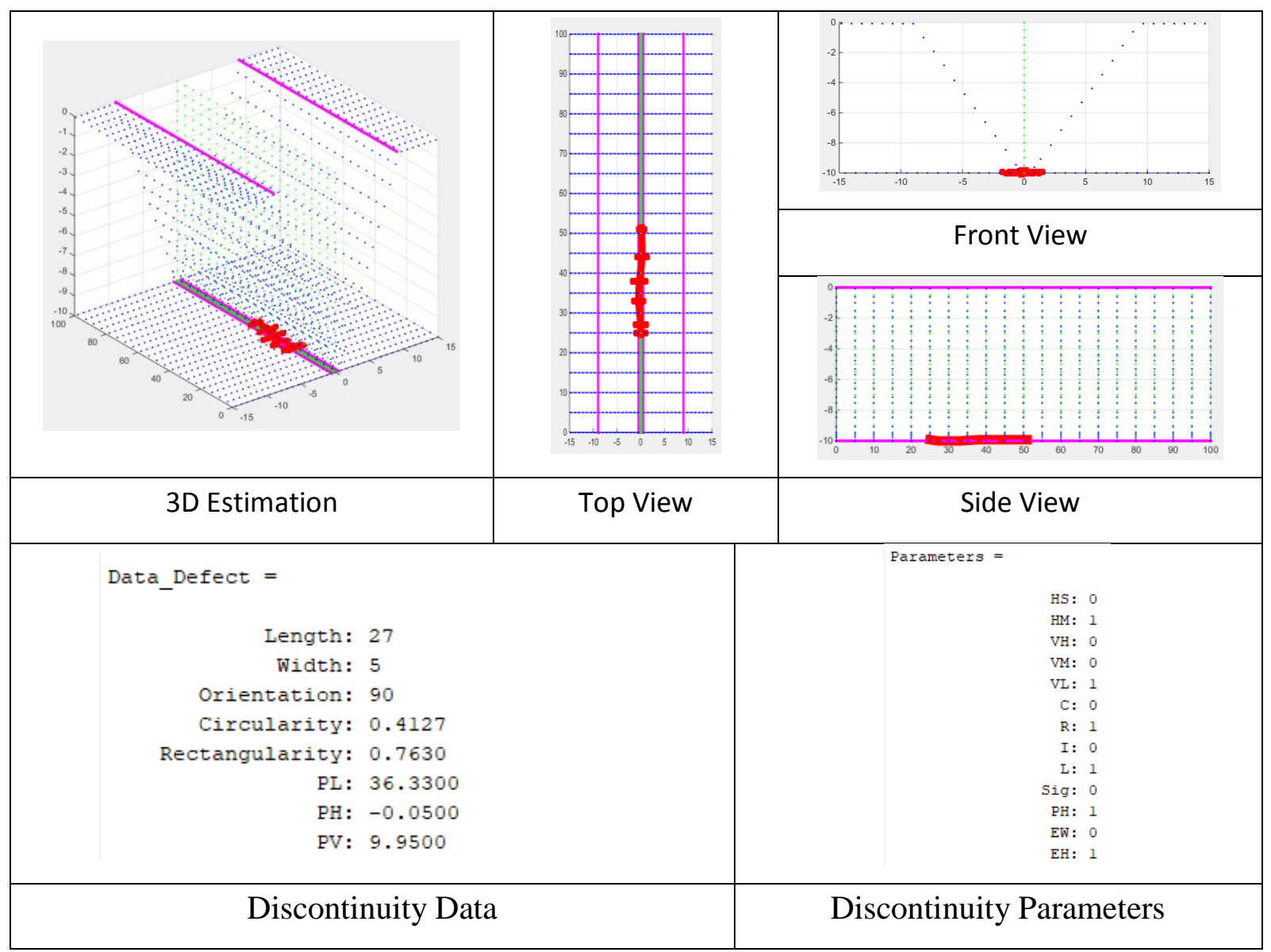

Fig.(12) MATLAP Program output of Lack of Pen. (LOP).

As shown into Fig. (12) above that MATLAP can characterize the LOP into 3D estimation as the photo taken above Fig.(10) at the same location and length.

\subsection{Longitudinal crack}

As shown in Fig.(13) that crack considering as longitudinal discontinuity at the weld filling and Fig.(14) shows different S-Scan images taken from X-Axis direction every $1 \mathrm{~mm}$ length position.

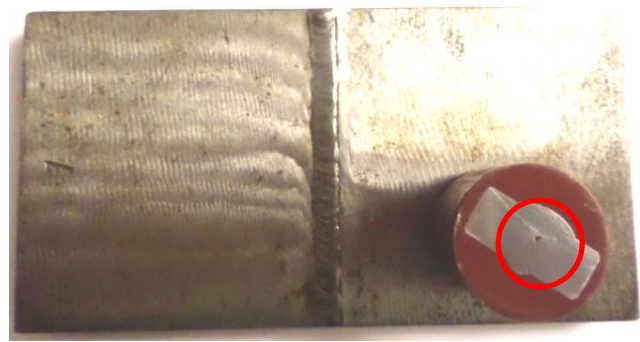

Fig.(13). Photo of LOP standard Discontinuity. 
Longitudinal Crack can be found even weld filling or sides, and it can be characterized as Longitudinal and rectangular shape, the standard division of discontinuity width was low, the echo width and height were large.
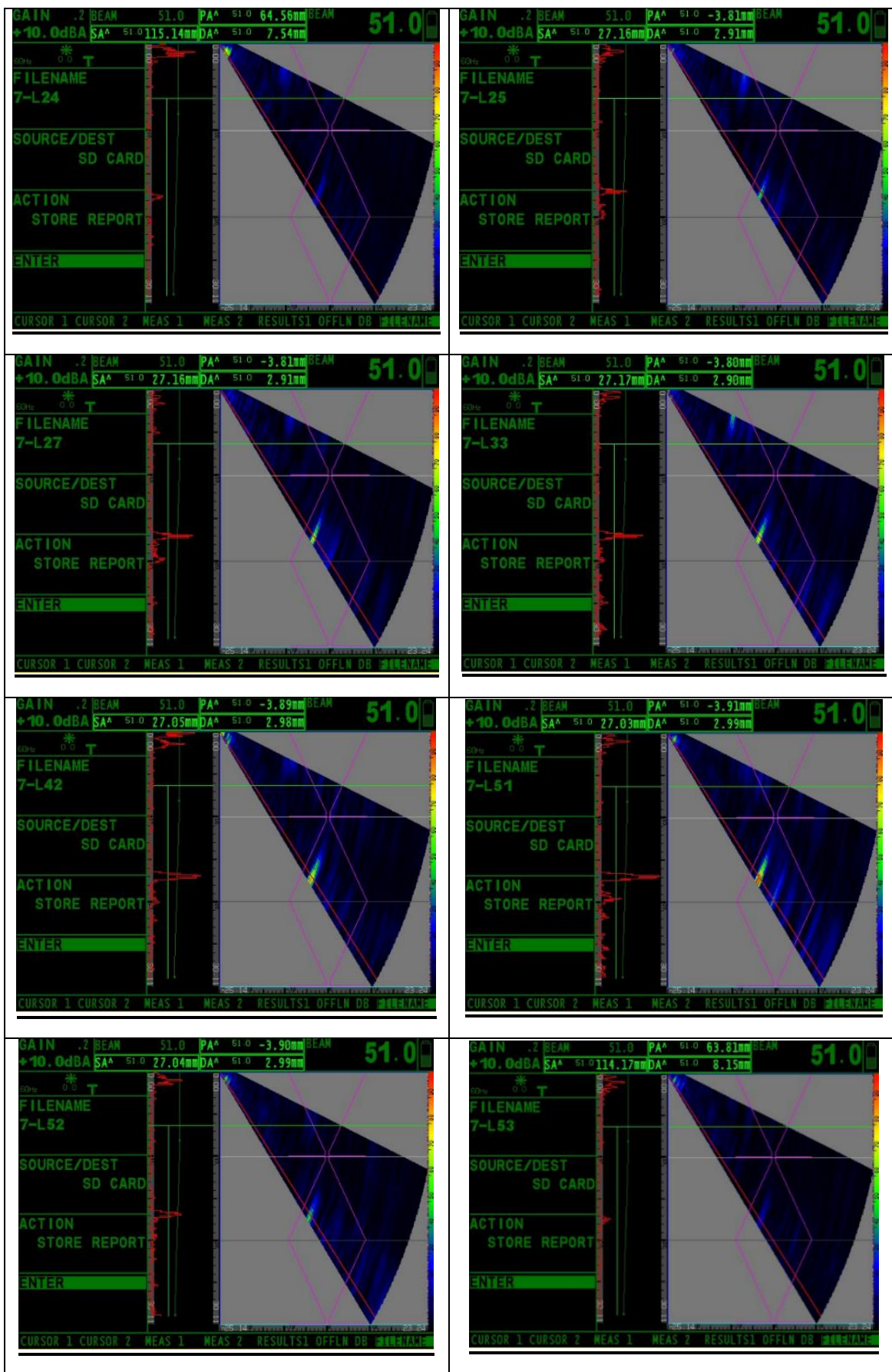

Fig.(14). Crack S-Scans taken respectively to be input to MATLAB Program. 


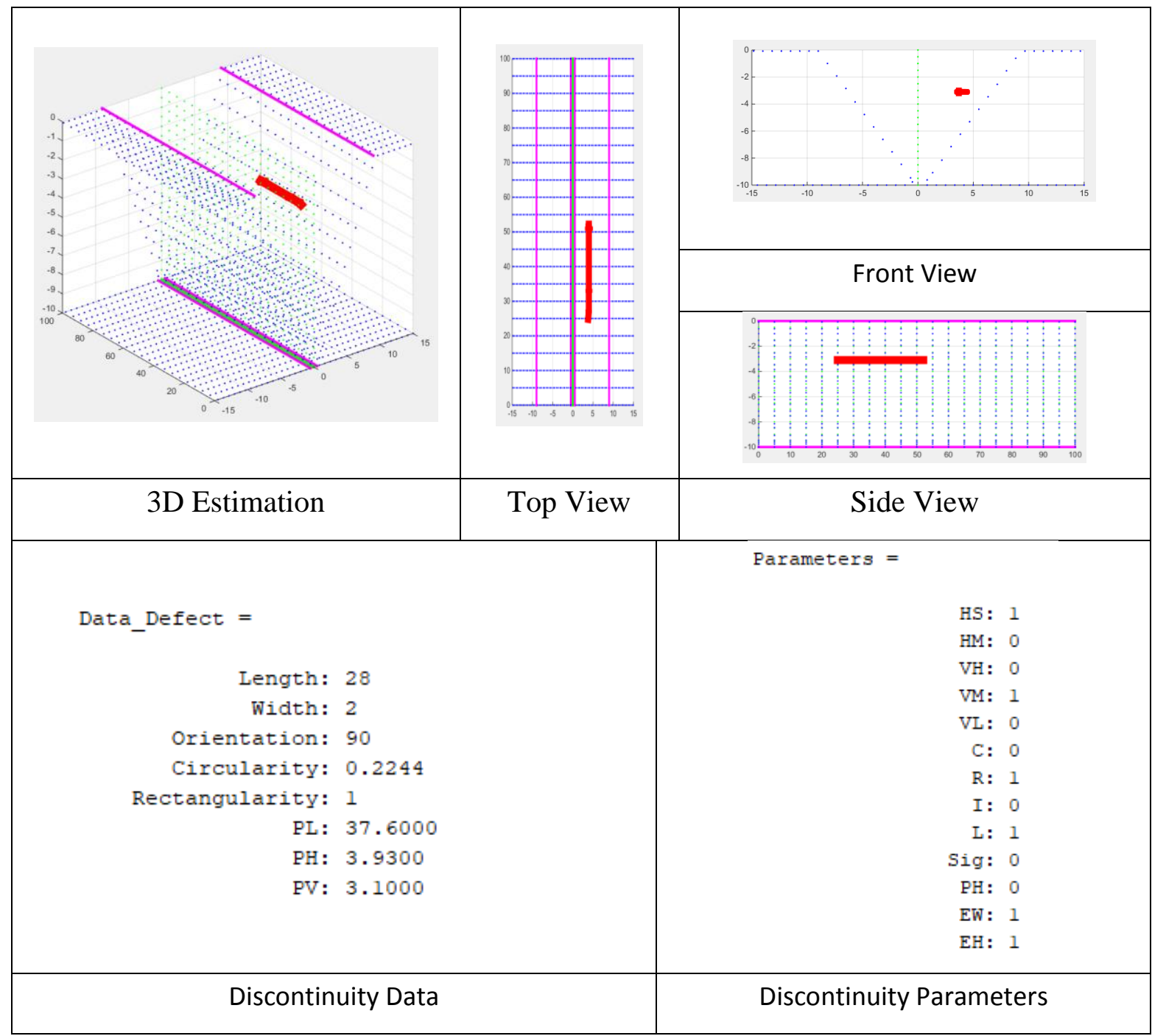

Fig.(15) MATLAP Program output Longitudinal Crack.

As shown into Fig.(15) above that MATLAP can characterize the crack as the photo taken above at the same location and it can be estimated the length which not shown at the photo Fig.(13).

\section{Flow Chart Procedure}

As shown in Fig.(16) it will enter S-Scan images to image processing to get location and Echo data $(\mathrm{PV}),(\mathrm{PH}),(\mathrm{PW}),(\mathrm{EH})$ and $(\mathrm{EW})$ then materialized to 3D Shape data (L), $(\mathrm{LW}),\left(A_{r}\right)\left(A_{\min }\right)$, Perimeter, $R_{f}$ and $F_{f}$. And calculate the parameters depend on the previous data. By using these parameters as inputs to ANN to characterize all types of welding discontinuities. 


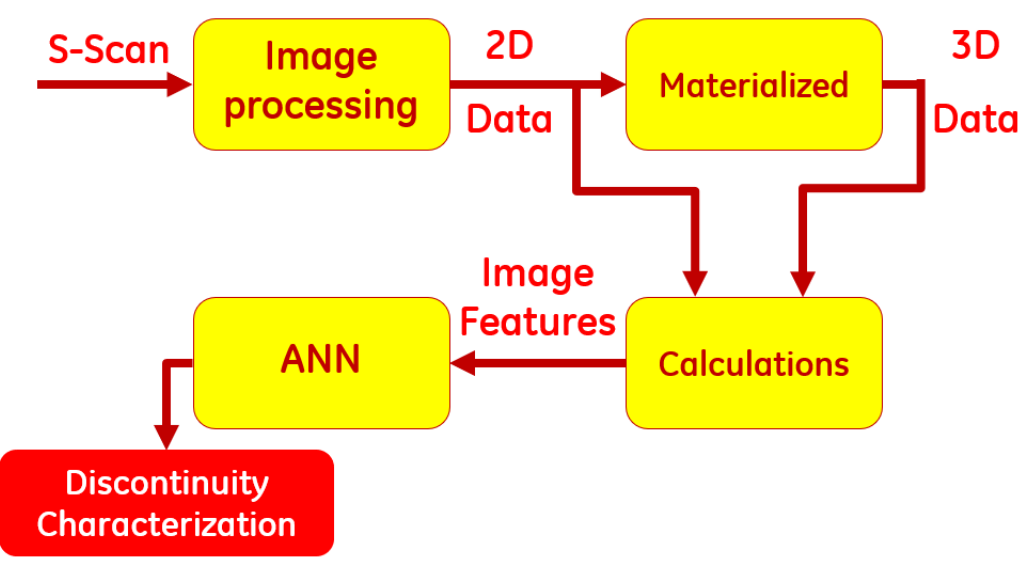

Fig.(16) Experimental Flow Chart Procedure.

\section{Artificial Neural Network Modeling}

\subsection{Estimation of learning input/output data}

1. Toe crack; can be found into side near to the weld cap, and it can be characterized as longitudinal and rectangular shape, the standard division of discontinuity width was low, the echo width and height were large.

2. Root crack; can be found around welding root, and it can be characterized as longitudinal and rectangular shape, the standard division of discontinuity width was low, the echo width and height were large.

3. Transverse crack; can be found into weld filling, and it can be characterized as transverse and rectangular shape, the standard division of discontinuity width was low, the echo width was large and the echo height was low.

4. Cluster Porosity; can be found at any location of weld, and it can be characterized as longitudinal and rectangular shape, but the standard division of discontinuity width was large, the echo width was large and the echo height was low.

5. Lack of Side Fusion; can be found at weld side, and it can be characterized as longitudinal and rectangular shape, but the standard division of discontinuity width was low, the echo width was low and the echo height was large (Sharp). 
6. Lack of Penetration; can be found exactly at the middle of weld root, and it can be characterized as longitudinal and rectangular shape, But the standard division of discontinuity width was low, the echo width was low and the echo height was large (Sharp).

7. Porosity; can be found at any location of weld, and it can be characterized as circular shape, But the standard division of discontinuity width was large, the echo width was large and the echo height was low.

8. Burn Through; can be found at any location, and it can be characterized as irregular shape, but the standard division of discontinuity width was high, the echo width and height were large.

9. Root Under Cut; can be found around welding root, and it can be characterized as longitudinal and rectangular or irregular shape, the standard division of discontinuity width was low, the echo width and height were low.

10. Longitudinal Crack; can be found even weld filling or sides, and it can be characterized as longitudinal and rectangular shape, the standard division of discontinuity width was low, the echo width and height were large.

11. Cap Undercut; can be found at weld cap, and it can be characterized as longitudinal and rectangular shape, the standard division of discontinuity width was low, the echo width and height were low.

12. Slag; If nothing appears from above estimations that characterize as Slag.

\subsection{Learning data}

From Table (3) below; it has 13-Inputs as parameters and 12-outputs as different types of discontinuities and if it interred the 13-different Inputs as above it will characterize the type of discontinuity.

\subsection{Perceptrons neural networks}

There are several types of NN but the Perceptrons NN is useful as classifier which is our focus. The design of a perceptron neural network is constrained completely by the problem to be solved. Perceptrons have a single layer of hard-limit neurons. The number of network inputs and the number of neurons in the layer are constrained by the number of inputs and outputs required by the problem. Fig.(18) shows the structure of proposed Perceptrons NN by entering 13-Inputs and 12-Outputs : 
Table (3). Learning Data as 13-Inputs (parameters and 12-outputs

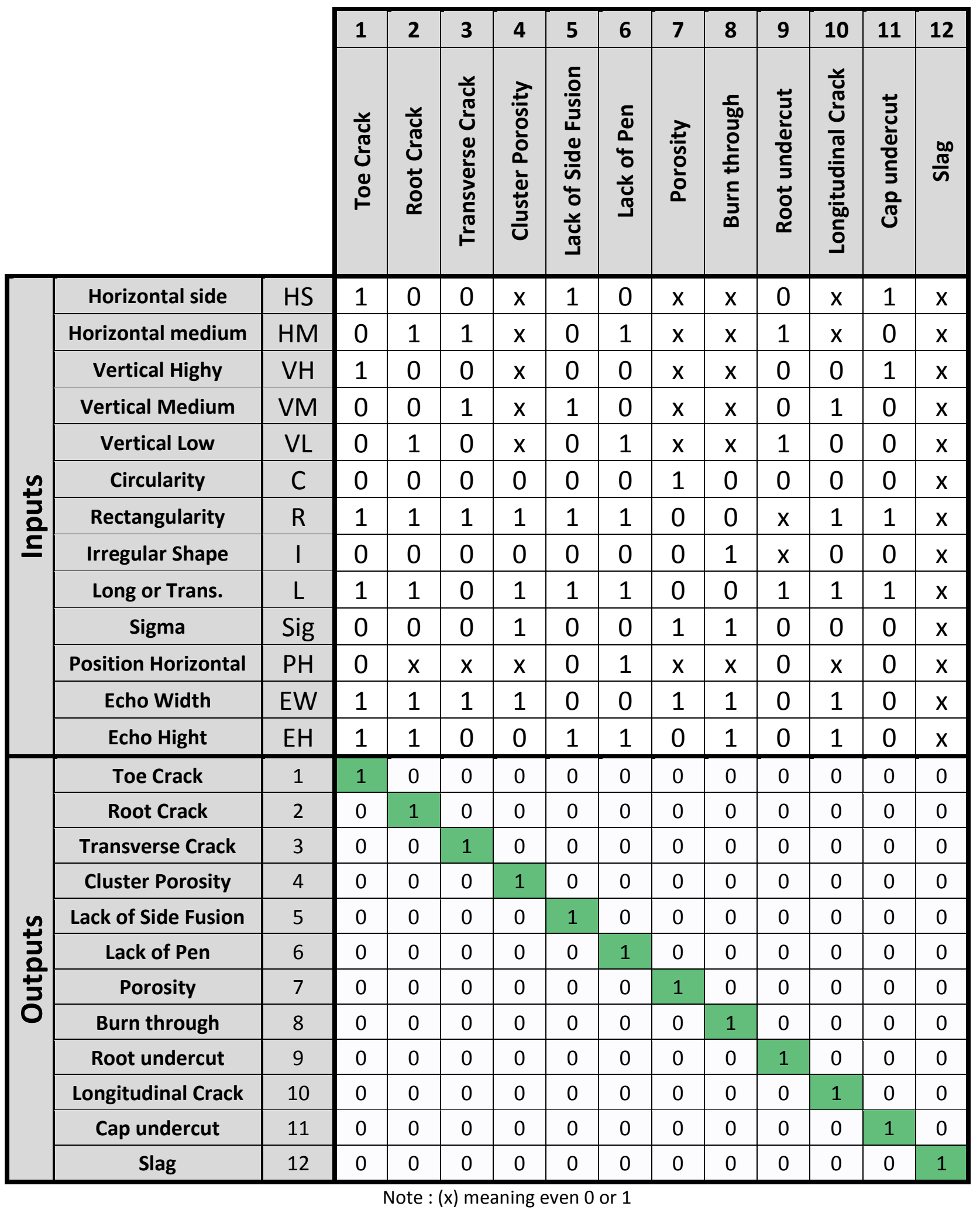




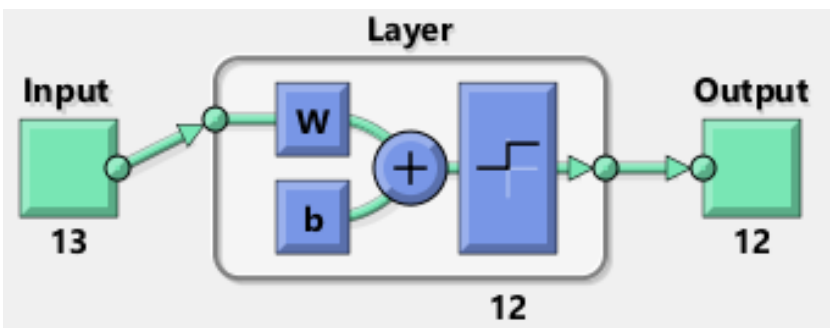

Fig.(18). Perceptrons ANN Input/Output Layers

\subsection{Practical data applied}

By applying the PACD system on both Groups (A and B) specimens. The system can easily characterize welding discontinuities into 12-types with zero error. Figs (19 and 20) show the classification of welding discontinuities (LOP and Cracks) respectively obtained from PACD system.

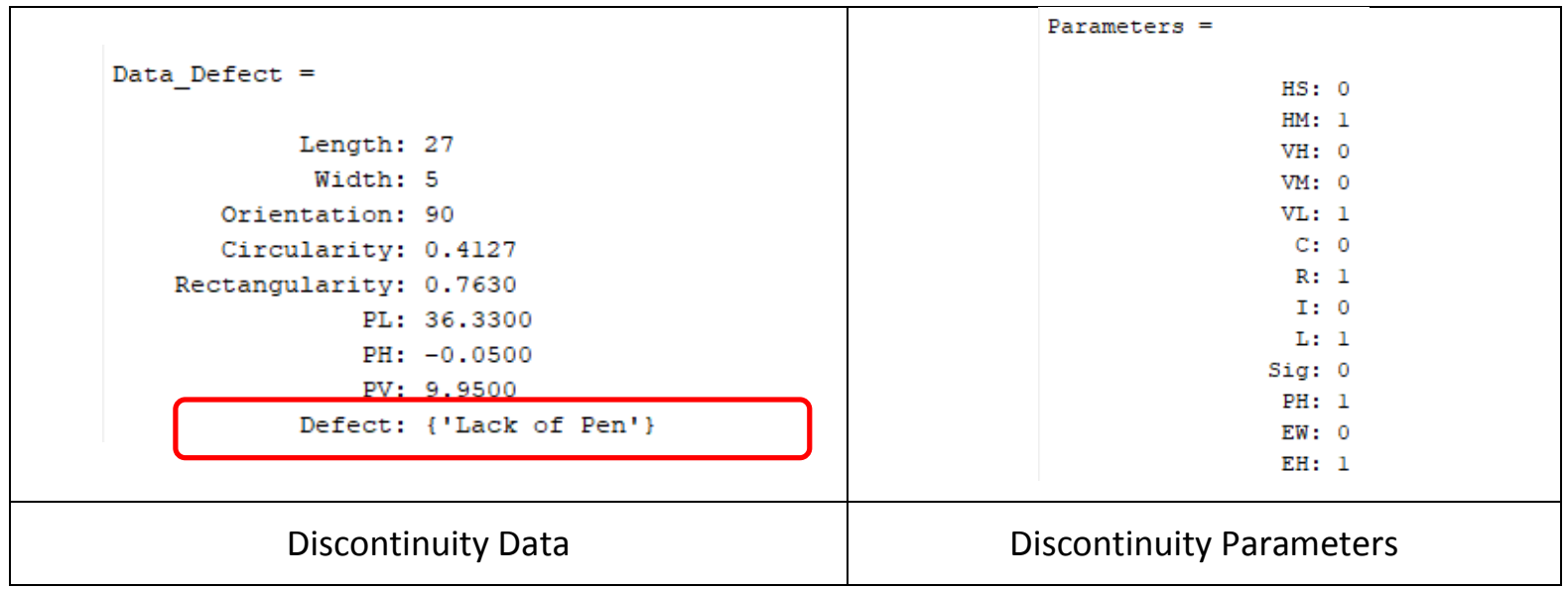

Fig.(19). Lack of Pen. (LOP) ANN Data Output.

\begin{tabular}{|r|r|}
\hline Data_Defect $=$ & Parameters $=$ \\
Length: 28 & HS: 1 \\
Width: $: 2$ & VH: 0 \\
Orientation: 90 & VM: 1 \\
Circularity : 0.2244 & VL: 0 \\
Rectangularity: 1 & C: 0 \\
PL: 37.6000 & R: 1 \\
PH: 3.9300 & I: 0 \\
PV: 3.1000 & L: 1 \\
\hline Defect: $\{$ 'Longtudinal Crack' & Sig: 0 \\
\hline & PH: 0 \\
\hline Discontinuity Data & EW: 1 \\
\hline
\end{tabular}

Fig.(20). Longitudinal Crack ANN Data Output. 


\section{Conclusions}

From the present work it can be concluded the following :

- Characterization of discontinuity type by using 3D-images is more reliable and accurate than 2D-images.

- ANN can solve arbitrarily complex welding discontinuities classification problem.

- Perceptron NN are capable easily classify 12-common welding discontinuities with Zero error.

- Collected data by using automatic detection (PACD) for welding discontinuity types were recorded and store the inspection continuous results of PA images to a data base system.

- Therefore, the reliability of testing and the precision of discontinuities evaluation were verified and depended less on the inspector's skill.

- PACD (A-Scan and S-Scan + ANN) can easily replace the Radiography method even (computerized and digital) advanced techniques.

\section{Future Work}

Currently; it must work in a project to apply and modify (PACD) in the interpretation and inspection of Ultrasonic Phased Array (PA) in the field. The aim of this work is to test and develop the PACD system so that it can be used to store the inspection results even by A-Scan and/or S-Scan to a database system. In addition, other information about the welder's performance will be stored to the database for further analysis and the common defects produced and its reason/s.

\section{Reference}

(1) Romeu R. Da Silva, Marcio H.S. Siqueira, "Estimated Accuracy of Classification of Defects Detected in Welded Joints by Radiographic Tests ", NDT\&E International, Vol.38, pp: 335-343, (2005).

(2) A. Hopgood, N. Woodcock, N. Hallam, "Interpreting Ultrasonic Images Using Rules, Algorithms and Neural Networks", European Journal, Vol.(2) No.(4), pp; 135-149, (2003).

(3) Obaidat, M.S. Suhail, Sadoun B., "Intelligent Simulation Methodology to Characterize Defects in Materials, Inf. Sci, Vol.(137), pp; 33-41, (2001). 
(4) Moura E., Silva R., Siqueira M., "Pattern Recognition of Weld Defects in Preprocessed TOFS Signals Using Linear Classifiers", Journal Nondestructive Evaluation , Vol.(23), pp; 163-172, (2004).

(5) Bettayeb F., Rachedi T., Benbartaoui H., "An improved Automated Ultrasonic NDE System by Wavelet and Neuron Networks", Ultrasonics, Vol.(43), pp; 853$858,(2004)$.

(6) Veiga J., Da Silva C., Rebello J., "The Use of Artificial Neural Network in the Classification of Pulse Echo and TOFD Ultrasonic Signals", Society of Mechanical and Scientific Engineering, Vol.(27), pp; 394-398, (2005).

(7) Min-jae Jung, Byeong-cheol Park, Jeong-hoon Bae, "PAUT Based Defect Detection Method for Submarine Pressure Hulls", International Journal for Naval Architecture and Ocean Engineering, Vol.(10), pp; 153-169, (2018). 